\title{
MiRNAs as Potential Prognostic Biomarkers for Metastasis in Thin and Thick Primary Cutaneous Melanomas
}

\author{
VIRGINIA VALENTINI ${ }^{1}$, VERONICA ZELLI ${ }^{1}$, EMANUELA GAGGIANO ${ }^{2}$, VALENTINA SILVESTRI ${ }^{1}$, \\ PIERA RIZZOLO ${ }^{1}$, AGOSTINO BUCALO $^{1}$, STEFANO CALVIERI $^{2}$, SARA GRASSI $^{2}$, PASQUALE FRASCIONE $^{3}$, \\ PIETRO DONATI ${ }^{4}$, GIUSEPPE SODA $^{1}$, LAURA OTTINI $^{1}$ and ANTONIO GIOVANNI RICHETTA ${ }^{2}$ \\ ${ }^{1}$ Department of Molecular Medicine, "Sapienza" University of Rome, Rome, Italy; \\ ${ }^{2}$ Department of Internal Medicine and Medical Specialties, \\ Unit of Dermatology, "Sapienza" University of Rome, Rome, Italy; \\ ${ }^{3}$ Department of Oncologic and Prevention Dermatology, \\ San Gallicano Dermatological Institute, IRCCS, Rome, Italy; \\ ${ }^{4}$ Laboratory of Cutaneous Histopathology, San Gallicano Dermatologic Institute, Rome, Italy
}

\begin{abstract}
Background/Aim: The identification of novel prognostic biomarkers for melanoma metastasis is essential to improve patient outcomes. To this aim, we characterized miRNA expression profiles in relation to metastasis in melanoma and correlated miRNAs expression with clinicalpathological factors. Materials and Methods: MiR-145-5p, miR-150-5p, miR-182-5p, miR-203-3p, miR-205-5p and miR$211-5 p$ expression levels were analyzed in primary cutaneous melanomas, including thin and thick melanomas, and in melanoma metastases by quantitative Real-Time PCR Results: A significantly lower miR-205-5p expression was found in metastases compared to primary melanomas. Furthermore, a progressive down-regulation of miR-205-5p expression was observed from loco-regional to distant metastasis. Significantly lower miR-145-5p and miR-203-3p expression levels were found in cases with Breslow thickness $>1 \mathrm{~mm}$, high Clark level, ulceration and mitotic rate $\geq 1 / \mathrm{mm}^{2}$. Conclusion: Our findings point to miR-205-5p as potential biomarker of distant metastases and to miR-145-5p and miR-203-3p as markers of aggressiveness in melanoma.
\end{abstract}

Cutaneous melanoma is increasing in incidence worldwide (1). In Italy, the incidence of cutaneous melanoma is about 5-7 cases $/ 100,000$ per year (2). From a clinical and biological standpoint, melanoma is a heterogeneous and unpredictable disease. In early detected melanoma, surgical excision remains

Correspondence to: Laura Ottini, Department of Molecular Medicine, "Sapienza" University of Rome, Viale Regina Elena, 324, Rome 00161, Italy. Tel: +39 0649918268, e-mail: laura.ottini@uniroma1.it

Key Words: Cutaneous melanoma, thin melanoma, thick melanoma, metastatic melanoma, metastasis, microRNAs, molecular biomarkers. the best choice of treatment and is curative in the vast majority of cases (3). However, when diagnosed at an advanced stage melanoma is metastatic (4) and, despite the scientific and technological advancements in the oncology field, there are currently no treatments capable of permanently treating patients with metastatic melanoma. For this reason, the most important strategy to reduce mortality is the early detection of the primary tumor and the identification of patients at highrisk of metastatic dissemination (5).

Main prognostic factors in primary melanomas include Breslow thickness, presence of ulceration, lymph node involvement and distant metastases (6). Breslow thickness provides the most useful histological information for prognosis in cutaneous melanoma.

Thin melanomas, which are lesions $\leq 1.00 \mathrm{~mm}$ in Breslow thickness, have an excellent prognosis (7). However, about $5-10 \%$ of patients with thin melanoma develop metastases, fatal in 5\% of the cases (7-9). Therefore, there is still controversy over the best way to use the clinical-pathological features to predict melanoma progression and metastasis development, and further molecular biomarkers are needed to identify cases with a high-risk of metastasis (10).

An increasing number of studies have shown that microRNAs (miRNAs), small (22 nucleotides) single-stranded non-coding RNAs that negatively regulate the expression of more than $60 \%$ of human genes (11), play an important role in different biological processes, including cell survival, apoptosis, cell cycle regulation and differentiation (12). MiRNA misregulation and the subsequent alteration of target gene expression may contribute to the development and progression of different types of cancer, including melanoma $(11,13)$. Several studies have underlined the high potential of miRNAs as diagnostic, prognostic and therapeutic markers in melanoma (14-16). 
To date, the majority of studies analyzing miRNA expression in melanoma have focused on the comparison between tumor and normal tissue, or between primary melanoma and metastases $(5,11,17-19)$. To our knowledge, the potential role of miRNAs as prognostic biomarkers of metastases in primary thin melanomas has not yet been evaluated.

In the present study, the expression profile of six miRNAs (miR-145, miR-150, miR-182, miR-203, miR-205, miR-211) involved in invasion and metastasis of melanoma $(5,11,19$ 22), were analyzed in primary cutaneous melanomas, including thin and thick melanomas, and in melanoma metastases in order to: identify possible differences in miRNA expression profile related to metastasis and distinguish patients at risk of metastases; correlate miRNAs expression with clinical-pathological factors.

\section{Materials and Methods}

Sample information. A series of 32 primary cutaneous melanoma cases, including 16 thin (Breslow thickness $\leq 1.00 \mathrm{~mm}$ ) and 16 thick (Breslow thickness $>1.00 \mathrm{~mm}$ ) melanomas, was analyzed in this study. Eight samples of metastatic specimens (four cutaneous, three lymph node and one lung metastases) were also analyzed. For three cases, matched primary tumor and metastatic specimens were available.

The series analyzed was retrospectively collected between April 2009 and April 2017 at the Department of Internal Medicine and Medical Specialties and Department of Radiology Oncology, and Anatomo-Pathology of Sapienza University of Rome and the Laboratory of Dermatopathology and Dermato-oncology and Preventive Unit of San Gallicano Institute of Rome.

All primary melanomas were characterized for the main clinicalpathological features, including age at diagnosis, sex, Breslow thickness, Clark level, presence of ulceration, tumor-infiltrating lymphocyte (TIL) grade based on assessment of absent, mild, moderate, or marked density (TIL grade 0 -III) and mitotic rate (number of mitoses $\left./ \mathrm{mm}^{2}\right)(23,24)$. Clinical follow-up ranged from 24 to 124 months (mean 63, median 48 months). For each study participant, sections of formalin-fixed paraffin-embedded (FFPE) tumor samples together with informed consent with a detailed description of the study protocol were obtained.

The study was approved by the Local Ethical Committee (Sapienza University of Rome, Prot. 873/13) and performed according to the Helsinki's declaration.

RNA extraction and miRNA expression analysis. For each sample, total RNA was extracted from 5-10 $\mu$ m FFPE tumor sections, using miRNeasy FFPE kit (Qiagen, Hilden, Germany) according to the manufacturer's instructions. Microdissection was applied to all samples to enrich in tumor cells. RNA quantity and quality were assessed using Agilent 2100 Bioanalyzer (Agilent Technologies, Santa Clara, CA, USA).

The expression profile of mature miRNAs for miR-145-5p, miR150-5p, miR-182-5p, miR-203-3p, miR-205-5p and miR-211-5p was investigated in primary melanoma samples and metastatic specimens by quantitative Real-Time PCR (qRT-PCR) using TaqMan Advanced miRNA Assays (Applied Biosystem, Carlsbad,
CA, USA). MiR-92a-3p, miR-103a-3p, miR-191-5p were used as endogenous controls for the normalization of miRNAs expression.

Reverse transcription was performed using TaqMan Advanced miRNA cDNA Synthesis Kit (Applied Biosystem) following manufacturer's protocol, and qRT-PCR was carried out on a 7500 Fast Real-Time PCR platform (Applied Biosystem). This method allows to quantify mature miRNAs with high sensitivity and specificity starting from $10 \mathrm{ng}$ of total RNA input.

Commercially available TaqMan assays for target and control miRNAs were used (Life Technologies). Each sample was run in triplicate and relative miRNA expression levels were determined by the $\Delta \mathrm{Ct}$ method (where $\Delta \mathrm{Ct}=\mathrm{Ct}_{\text {target gene }}-\mathrm{Ct}_{\text {mean of the endogenous controls }}$ ) and expressed as $2^{-} \Delta \mathrm{Ct}$. Appropriate negative controls were also included in each experiment.

Statistical analysis. Unsupervised hierarchical clustering analysis was performed to evaluate relevant clusters and co-expression. Dendrograms and heatmap were generated using Euclidean distance matrix and complete linkage.

The non-parametric Mann-Whitney-Wilcoxon test was used to compare relative miRNA expression levels for each miRNA in primary non-metastatic melanomas, primary metastatic melanomas and metastatic specimens.

Associations between miRNA expression levels and clinicalpathological characteristics were evaluated using linear regression analysis, and the non-parametric Mann-Whitney-Wilcoxon or Kruskal-Wallis test, where appropriate. A $p$-value $\leq 0.05$ was considered statistically significant. All statistical analyses were performed with the R software (www.r-project.org).

\section{Results}

MiRNA expression analysis in primary melanomas and melanoma metastases. A series of 32 primary cutaneous melanoma cases, comprising 16 thin and 16 thick melanomas and including 17 non-metastatic and 15 metastatic cases, was analyzed (Figure 1). Eight samples of metastatic specimens, including three samples with matched primary tumor, were also examined.

As shown in Table I, the mean age at melanoma diagnosis was 59.3 years (range $=18-92$ years) and a comparable number of male and female individuals $(53.1 \%$ and $46.9 \%$, respectively) were included in the study. In the vast majority melanoma cases showed Clark level III (46.9\%), absence of ulceration $(81.2 \%)$ and TIL grade I-II $(71.9 \%)$. A mitotic rate $<1 / \mathrm{mm}^{2}$ and $\geq 1 / \mathrm{mm}^{2}$ was detected in $53.1 \%$ and $46.9 \%$ of cases, respectively.

Overall, expression levels of miR-145-5p, miR-150-5p, miR-182-5p, miR-203-3p, miR-205-5p and miR-211-5p were examined in 40 samples (32 primary melanomas and eight metastases).

Unsupervised hierarchical clustering analysis identified two principal groups of clustered miRNAs: one group including miR-211-5p, miR-150-5p and miR-145-5p, and the other group including miR-205-5p, miR-203-3p and miR182-5p (Figure 2). No clear distinctive clusters of melanoma cases in relation to metastatic status emerged. 


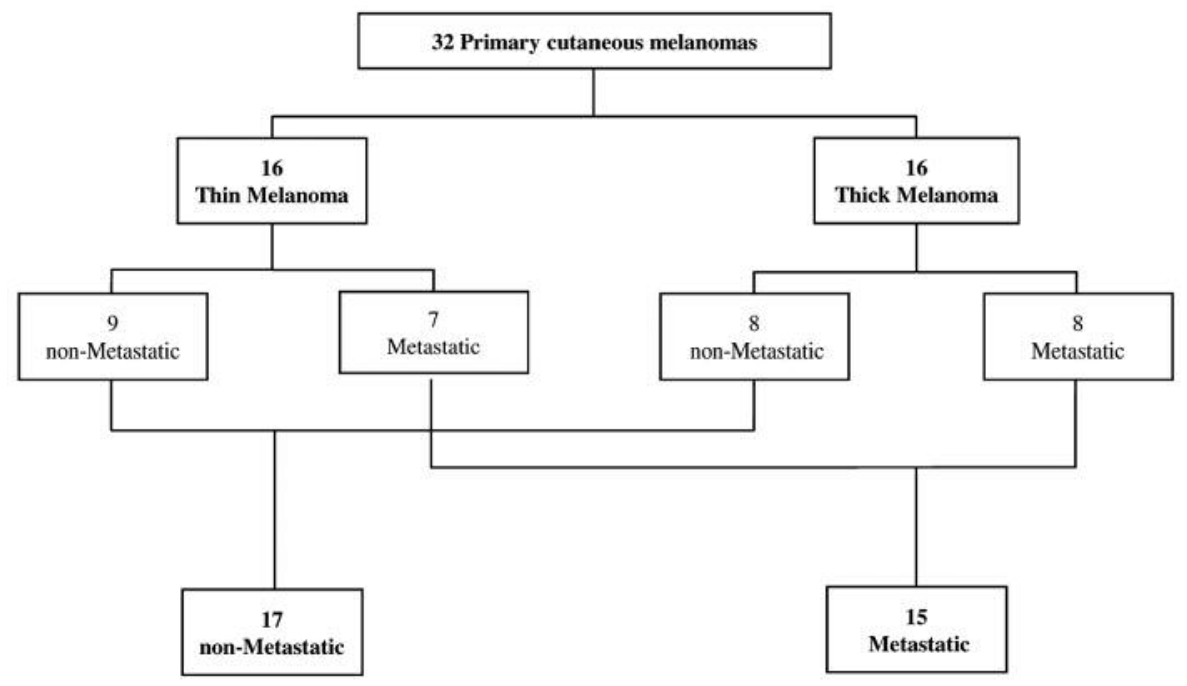

Figure 1. Diagram showing the distribution of 32 primary cutaneous melanoma cases analyzed in this study. Thin and Thick refers to melanomas with Breslow thickness $\leq 1 \mathrm{~mm}$ and $>1 \mathrm{~mm}$, respectively.

By comparing miRNA expression levels among primary non-metastatic melanomas, primary metastatic melanomas and metastases, statistically significant differences emerged in the expression levels of miR-145-5p, miR-203-3p and miR-205-5p (Figure 3). In particular, miR-145-5p and miR203-3p showed decreased expression levels in metastases compared with primary non-metastatic tumors $(p=0.05$ and $p=0.02$, respectively), whereas $\operatorname{miR}-205-5 p$ showed significantly lower expression levels in metastases compared with primary metastatic melanomas $(p=0.04)$.

MiR-205-5p expression was further compared in the three cases of primary metastatic melanoma and their corresponding metastasis (Figure 4a). The difference in miR205-5p expression between primary tumor and corresponding metastasis varied in relation to the site of metastasis. In particular, miR-205-5p expression was 6- and 54-fold higher in the primary melanomas compared with the relative lymph node and lung metastasis, respectively. MiR205-5p expression was also examined in all eight metastatic specimens according to the metastatic site (Figure 4b). Overall, a progressive down-regulation of miR-205-5p expression was observed from loco-regional metastasis (cutaneous and lymph node metastasis) to distant metastasis (lung metastasis).

Possible differences in miRNAs expression levels among primary tumors were also investigated considering thin and thick melanomas separately. No statistically significant differences in the expression levels of the six miRNAs analyzed emerged. However, the expression levels of miR145-5p, miR-150-5p and miR-205-5p showed an opposite trend in non-metastatic and metastatic cases when comparing
Table I. Clinical-pathological characteristics of the 32 primary cutaneous melanoma cases analyzed in this study.

\begin{tabular}{lc}
\hline Clinical-pathological characteristics & $\mathrm{N}(\%)$ \\
\hline Mean age at diagnosis \pm SD (range) & $59.3 \pm 20.1(18-92)$ \\
Gender & \\
Male & $17(53.1 \%)$ \\
Female & $15(46.9 \%)$ \\
Breslow thickness & \\
$\leq 1 \mathrm{~mm}$ (thin) & $16(50.0 \%)$ \\
$>1 \mathrm{~mm}$ (thick) & $16(50.0 \%)$ \\
Clark level & \\
II & $2(6.2 \%)$ \\
III & $15(46.9 \%)$ \\
IV & $9(28.1 \%)$ \\
V & $6(18.7 \%)$ \\
Ulceration & \\
Absent & $26(81.2 \%)$ \\
Present & $6(18.7 \%)$ \\
TIL grade & \\
0 & $6(18.7 \%)$ \\
I & $11(34.4 \%)$ \\
II & $12(37.5 \%)$ \\
III & $3(9.4 \%)$ \\
Mitotic rate $\left(\mathrm{N} / \mathrm{mm}^{2}\right)$ & \\
$<1 / \mathrm{mm}^{2}$ & $17(53.1 \%)$ \\
$\geq 1 / \mathrm{mm}^{2}$ & $15(46.9 \%)$ \\
\hline
\end{tabular}

N: Number of cases; SD: standard deviation; TIL: Tumor-infiltrating lymphocyte; $\left(\mathrm{N} / \mathrm{mm}^{2}\right)$ : number of mitoses $/ \mathrm{mm}^{2}$.

thin and thick melanomas (Figure 5). Furthermore, when considering median expression levels in thin and thick melanomas in relation to metastatic status, higher median 


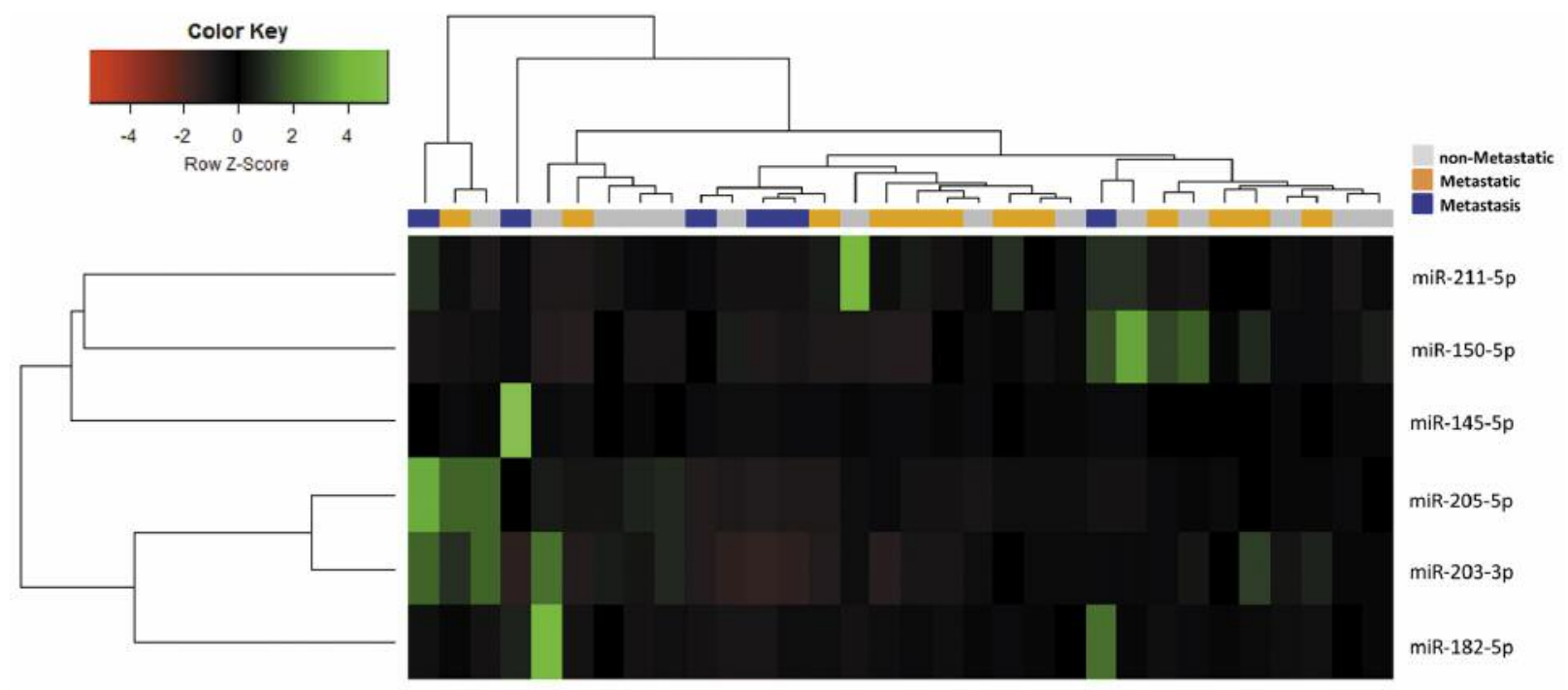

Figure 2. Unsupervised hierarchical clustering analysis of relative expression levels of target miRNAs in 32 samples (including 14 primary nonmetastatic melanomas, 12 primary metastatic melanomas and 6 metastases), for which data on expression levels of all miRNAs examined were available.

expression levels of miR-145-5p, miR-182-5p, miR-203-3p and miR-205-5p were observed in metastatic thin melanomas compared with non-metastatic thick melanomas. In particular, for miR-203-3p, a progressive decrease in expression levels was observed from thin non-metastatic to thick metastatic melanomas.

Association between miRNA expression and clinicalpathological characteristics of primary melanomas. For each miRNA, association between expression levels and clinicalpathological characteristics of the 32 primary melanoma cases was evaluated (Table II). Overall, a significant association between lower expression of miR-145-5p and miR-203-3p and pathological characteristics indicative of aggressiveness was observed. In particular, significantly lower miR-145-5p and miR-203-3p expression levels were found in cases with Breslow thickness $>1 \mathrm{~mm}(p=0.002$ and $p=0.005$, respectively), high Clark level $(p=0.007$ and $p=0.005$, respectively), ulceration $(p=0.00001$ and $p=0.0002$, respectively) and mitotic rate $\geq 1 / \mathrm{mm}^{2}(p=0.02$ and $p=0.001$ respectively). Significantly lower miR-182-5p expression levels were also observed in cases with Breslow thickness $>1 \mathrm{~mm}(p=0.03)$ and high Clark level $(p=0.03)$.

\section{Discussion}

In this study, we explored the possible role of miRNAs, known to be involved in the metastatic process, as prognostic biomarkers for metastasis in melanoma. To this aim, the expression profile of mature miRNAs for miR-145-5p, miR- 150-5p, miR-182-5p, miR-203-3p, miR-205-5p and miR211-5p was investigated in primary non-metastatic melanomas, primary metastatic thin and thick melanomas and in metastases.

In line with previously reported findings, our study showed lower expression levels of miR-145-5p, miR-203-3p and miR-205-5p in metastases compared to primary tumors, highlighting their role in metastasis (16, 25-29).

In particular, our results indicate significantly lower miR205-5p expression in metastases compared with primary metastatic melanomas. Comparing the expression of miR205-5p between primary metastatic melanomas and their corresponding metastases, the ratio (primary/metastasis) was found to higher with increasing metastases distance. In fact, the evaluation of miR-205-5p in metastases according to their location, showed a progressive decrease in expression from loco-regional (cutaneous and lymph node) to distant (lung) metastases, thus suggesting that the down-regulation of miR-205-5p expression might promote the development of distant metastases. These results are consistent with recent findings showing a progressive loss of miR-205-5p along the full spectrum of melanoma progression, from primary tumor to distant metastasis (19).

Several studies suggest that miR-205-5p is a tumor suppressor miRNA in melanoma and has a role in the regulation of melanoma cell invasiveness $(19,30)$, mainly through the regulation of zinc-finger E-box binding homeobox 2 (ZEB2) and E-cadherin expression (31). However, although the role of miR-205-5p as tumor suppressor in melanoma is well established, its use as 

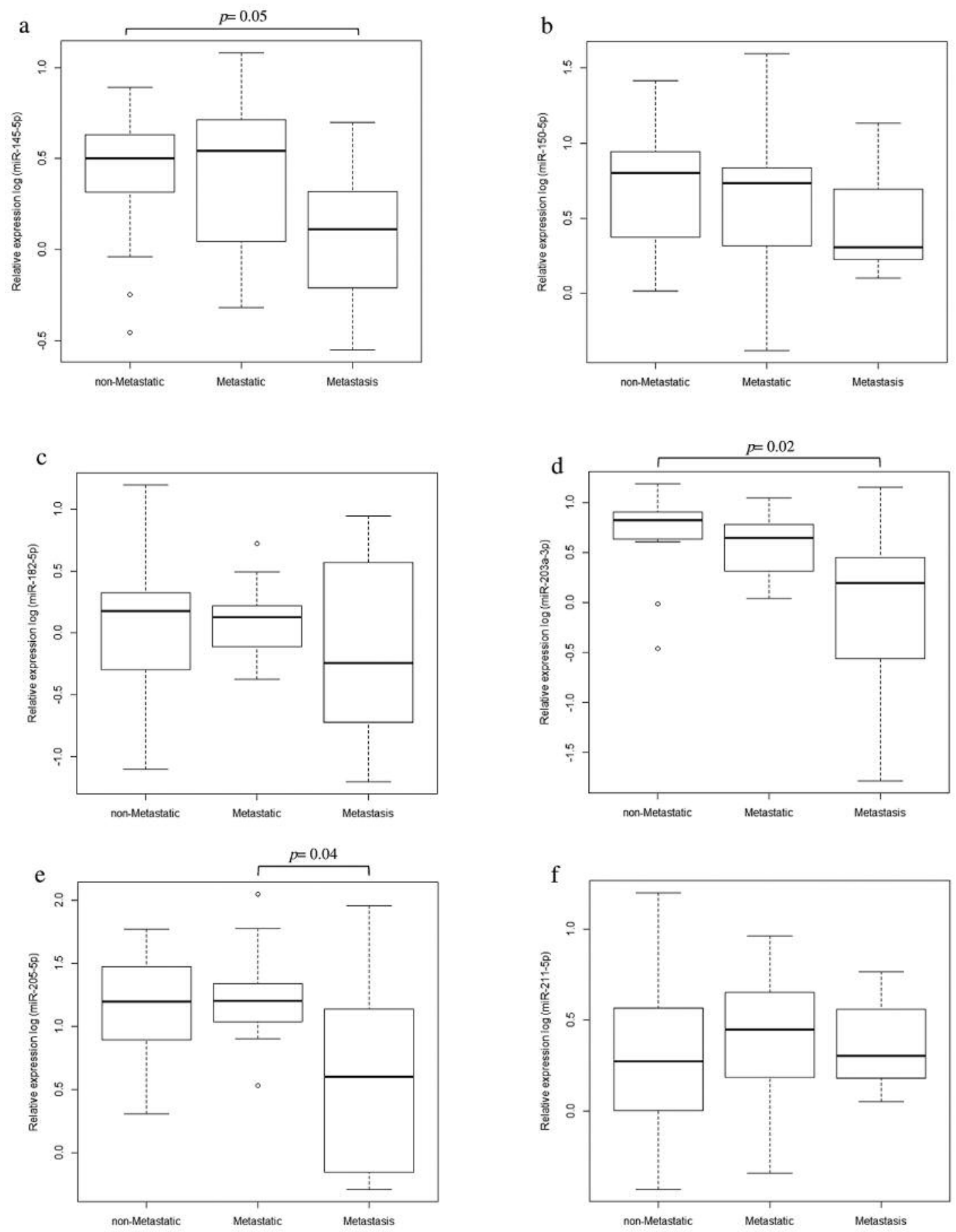

Figure 3. Comparison of relative expression levels of miR-145-5p (a), miR-150-5p (b), miR-182-5p (c), miR-203-3p (d), miR-205-5p (e) and miR$211-5 p(f)$ in samples of primary non-metastatic melanomas, metastatic melanomas and metastases. Results of miRNA expression analysis are shown as log values in boxplots. Statistically significant p-values are reported.

molecular biomarker has not yet been validated. Overall, our findings add evidence supporting the utility of miR-205-5p as a prognostic marker in melanoma, particularly for the selection of patients at risk of developing distant metastases.
In our study, lower miR-145-5p and miR-203-3p expression levels in primary melanomas were significantly correlated with pathological characteristics suggestive of aggressiveness, including Breslow thickness $>1 \mathrm{~mm}$, high Clark level, 
a

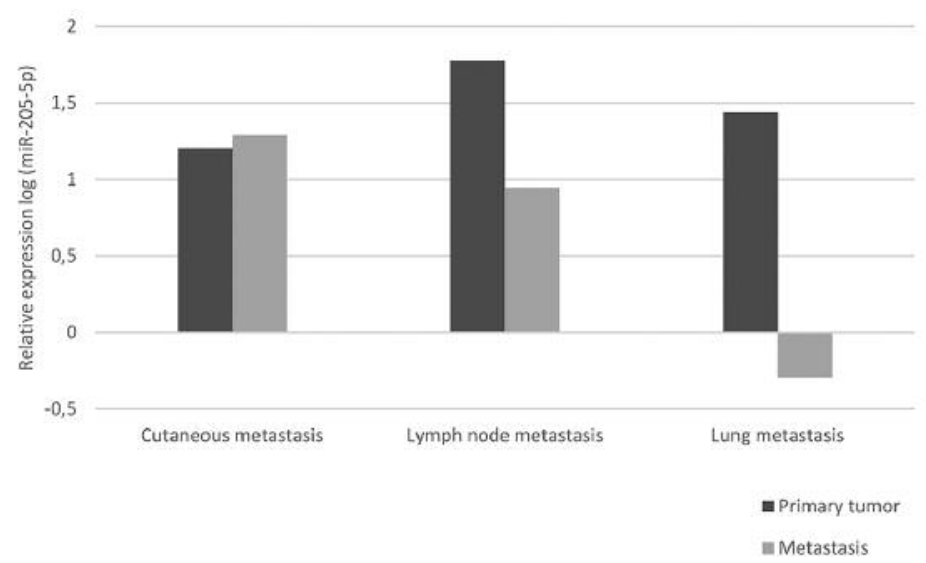

b

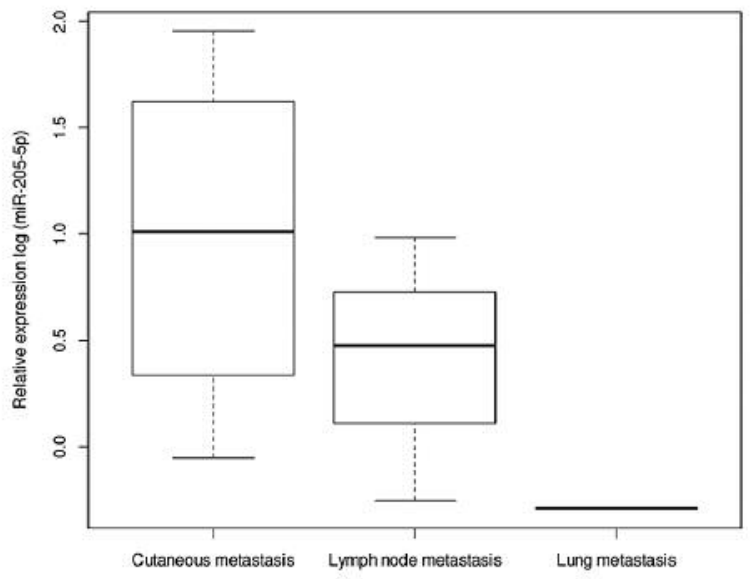

Figure 4. Relative expression levels of miR-205-5p in the three primary metastatic tumors and their corresponding metastasis (a) and in all the metastatic specimens available $(N=8)$, stratified according to metastatic site $(b) . * m i R-205-5 p$ expression ratio calculated as primary tumor versus corresponding metastasis.

Table II. Associations between relative miRNA expression levels and clinical-pathological characteristics in the 32 primary cutaneous melanoma cases analyzed in this study.

\begin{tabular}{|c|c|c|c|c|c|c|}
\hline & miR-145-5p & miR-150-5p & miR-182-5p & miR-203-3p & miR-205-5p & miR-211-5p \\
\hline & Median & Median & Median & Median & Median & Median \\
\hline \multicolumn{7}{|l|}{ Gender } \\
\hline Male & 2.98 & 6.31 & 1.40 & 5.78 & 16.20 & 2.10 \\
\hline Female & 3.73 & 4.10 & 1.65 & 5.50 & 15.80 & 2.70 \\
\hline$p$-value & 0.82 & 0.35 & 0.82 & 0.31 & 0.31 & 0.33 \\
\hline \multicolumn{7}{|l|}{ Breslow thickness } \\
\hline$\leq 1 \mathrm{~mm}($ thin $)$ & 4.24 & 6.06 & 1.64 & 7.03 & 16.44 & 1.96 \\
\hline$>1 \mathrm{~mm}$ (thick) & 1.41 & 3.18 & 0.81 & 3.55 & 12.71 & 3.67 \\
\hline$p$-value & 0.002 & 0.51 & $\mathbf{0 . 0 3}$ & 0.005 & 0.30 & 0.17 \\
\hline \multicolumn{7}{|l|}{ Clark level } \\
\hline II & 3.85 & 1.70 & 9.31 & 12.70 & 34.1 & 1.49 \\
\hline III & 4.56 & 6.08 & 1.60 & 6.52 & 15.8 & 2.04 \\
\hline IV & 1.39 & 1.66 & 0.61 & 2.36 & 8.20 & 4.46 \\
\hline $\mathrm{V}$ & 1.17 & 7.01 & 1.22 & 4.40 & 37.63 & 1.62 \\
\hline$p$-value & 0.007 & 0.12 & 0.03 & 0.005 & 0.06 & 0.39 \\
\hline \multicolumn{7}{|l|}{ Ulceration } \\
\hline Absent & 3.62 & 5.76 & 1.55 & 6.45 & 16.44 & 2.51 \\
\hline Present & 0.80 & 4.04 & 0.61 & 1.60 & 9.01 & 1.52 \\
\hline$p$-value & 0.00001 & 0.55 & 0.13 & 0.0002 & 0.07 & 0.45 \\
\hline \multicolumn{7}{|l|}{ TIL grade } \\
\hline 0 & 3.89 & 4.79 & 1.23 & 3.50 & 13.60 & 2.04 \\
\hline I & 2.11 & 2.57 & 1.47 & 6.66 & 16.20 & 2.45 \\
\hline II & 3.53 & 6.93 & 1.26 & 5.01 & 15.02 & 2.20 \\
\hline III & 3.73 & 8.88 & 2.11 & 6.52 & 19.90 & 2.93 \\
\hline$p$-value & 0.78 & 0.07 & 0.36 & 0.33 & 0.89 & 0.94 \\
\hline \multicolumn{7}{|c|}{ Mitotic rate $\left(\mathrm{N} / \mathrm{mm}^{2}\right)$} \\
\hline$<1 / \mathrm{mm}^{2}$ & 3.89 & 6.31 & 1.60 & 6.79 & 16.20 & 2.45 \\
\hline$\geq 1 / \mathrm{mm}^{2}$ & 1.43 & 5.41 & 0.81 & 2.68 & 11.36 & 2.16 \\
\hline$p$-value & 0.02 & 0.31 & 0.06 & 0.001 & 0.45 & 0.86 \\
\hline
\end{tabular}

Bold $p$-values are considered statistically significant. TIL: Tumor-infiltrating lymphocyte; $\left(\mathrm{N} / \mathrm{mm}^{2}\right)$ : number of mitoses $/ \mathrm{mm}^{2}$. 

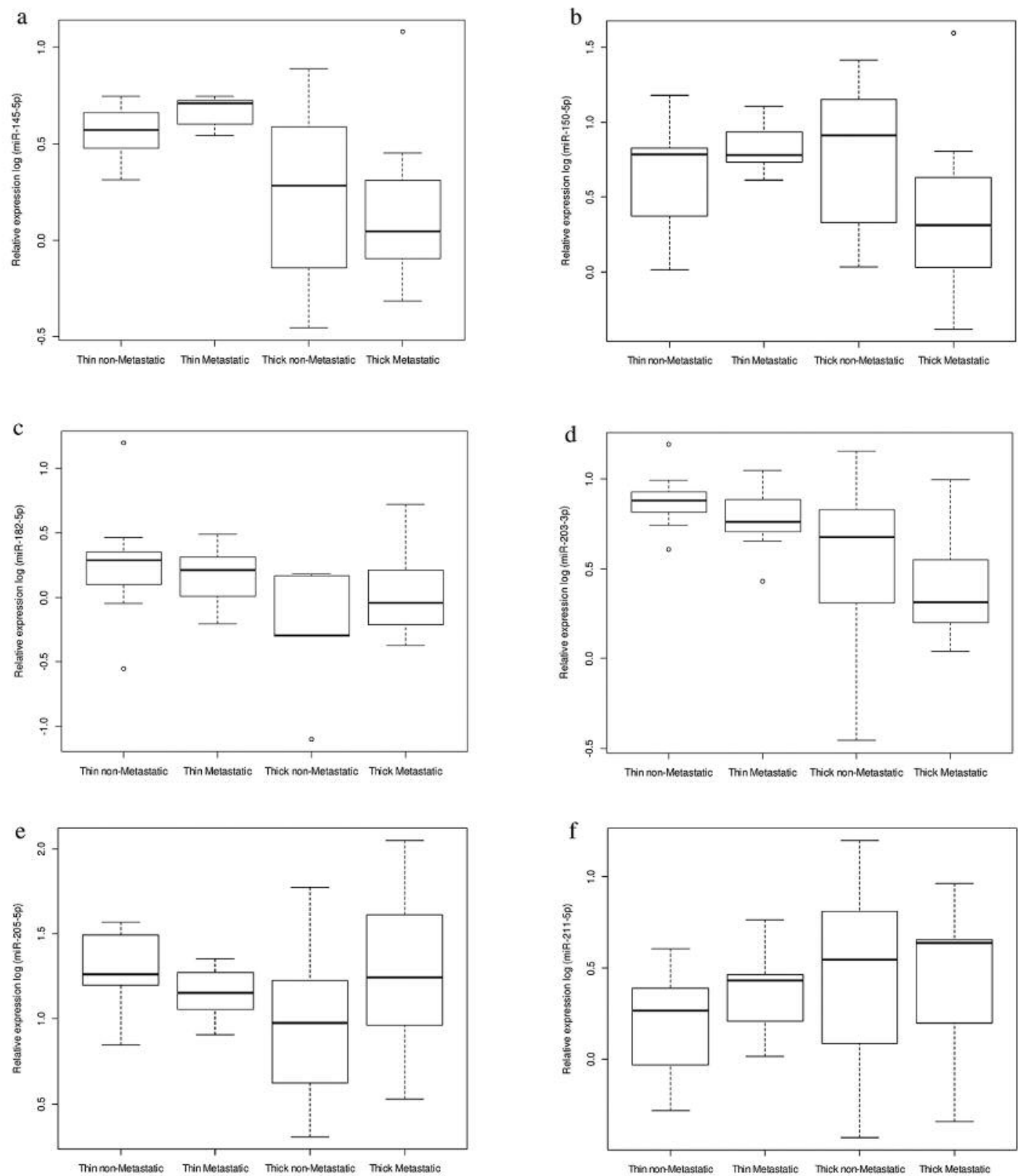

Figure 5. Comparison of relative expression levels of miR-145-5p (a), miR-150-5p (b), miR-182-5p (c), miR-203-3p (d), miR-205-5p (e) and miR$211-5 p(f)$ in primary thin and thick melanomas stratified according to metastatic behavior. Results of miRNA expression analysis are shown as log values in boxplots.

ulceration and mitotic rate $\geq 1 / \mathrm{mm} 2$. MiR-145-5p and miR203-3p play a key role as tumor suppressor miRNAs and both miRNAs were down-regulated in melanoma (20). MiR-145$5 p$ inhibits the invasive and metastatic capacity of neoplastic cells by repressing fascin homolog 1 (FSCN1) $(21,25)$ and the ectopic expression of miR-145-5p in melanoma cell lines, resulting in a decrease in cell growth and invasiveness through targeting c- MYC (29). By repressing different target genes, a similar suppressive effect was observed for miR-203-3p (27, 28). In particular, miR-203-3p inhibited the proliferation through targeting B lymphoma Mo-MLV insertion region 1 homolog (BMI1) (32) and the ectopic expression of miR- 203$3 p$ in melanoma cells reduced the expression of E2F3a and E2F3b, leading to the inhibition of cell growth (29). Taking into account these observations, our findings suggest that in primary melanomas, miR-145-5p and miR-203-3p may be 
involved in the regulation of tumor growth and invasion. Further studies are needed in order to support the clinical relevance of miR145-5p and miR-203-3p expression as prognostic biomarkers for metastatic melanomas.

We also showed that the stratification of miR-145-5p expression according to Breslow thickness and metastatic behavior highlighted an opposite trend in the expression levels of this miRNA between non-metastatic and metastatic cases in thin compared to thick melanomas. This tendency was also observed for miR-150-5p and miR-205-5p. These results suggest a molecular heterogeneity in the metastatic process in relation to thickness and may indicate that different molecular mechanisms could mediate disease progression and metastasis development in thin compared to thick melanomas. To date, only few studies have investigated the molecular processes underlying thin melanoma pathogenesis and no molecular predictor of disease progression has been identified (30, 3335). Overall, our results add evidence supporting the hypothesis that thin melanoma cannot be considered just an early stage of melanoma progression but, at least in some cases, a different type of tumor (33).

In conclusion, our findings add insights into the characterization of miRNAs expression profile of thin and thick melanomas, pointing to miR-205-5p as a potential biomarker of distant metastases and to miR-145-5p and miR203-3p as biomarkers of aggressiveness in primary tumors.

\section{Conflicts of Interest}

The Authors declare no conflicts of interest regarding this study.

\section{Authors' Contributions}

VV: Performed experiments and statistical analyses, interpreted the results and drafted the manuscript. VZ and EG: performed experiments and interpreted the results. VS, PR and $\mathrm{AB}$ : performed statistical analyses and interpreted the results. SC, SG, PF, PD and GS: recruited samples and collected clinical-pathological data. LO and AGR: conceived, designed and coordinated the study and drafted the manuscript. All authors reviewed, edited and approved the manuscript for publication.

\section{Acknowledgements}

The study was partially supported by the Italian Association for Cancer Research (AIRC-IG21389) to Laura Ottini. The Authors thank all the patients who participated in this study and the institutions and their staff who supported the recruitment of patients and the collection of samples and data.

\section{References}

1 Siegel RL, Miller KD and Jemal A: Cancer statistics, 2019. CA Cancer J Clin 69(1): 7-34, 2019. PMID: 30620402. DOI: $10.3322 /$ caac. 21551
2 Rastrelli M, Tropea S, Rossi CR and Alaibac M: Melanoma: epidemiology, risk factors, pathogenesis, diagnosis and classification. In Vivo 28(6): 1005-1011, 2014. PMID: 25398793.

3 Ross MI and Gershenwald JE: Evidence-based treatment of early-stage melanoma. J Surg Oncol 104(4): 341-353, 2011. PMID: 21858828. DOI: $10.1002 /$ jso. 21962

4 Garbe C, Peris K, Hauschild A, Saiag P, Middleton M, Bastholt L, Grob JJ, Malvehy J, Newton-Bishop J, Stratigos AJ, Pehamberger $\mathrm{H}$ and Eggermont AM; European Dermatology Forum (EDF); European Association of Dermato-Oncology (EADO); European Organisation for Research and Treatment of Cancer (EORTC): Diagnosis and treatment of melanoma. European consensus-based interdisciplinary guideline - Update 2016. Eur J Cancer 63: 201217, 2016. PMID: 27367293. DOI: 10.1016/j.ejca.2016.05.005

5 Ross CL, Kaushik S, Valdes-Rodriguez R and Anvekar R: MicroRNAs in cutaneous melanoma: Role as diagnostic and prognostic biomarkers. J Cell Physiol 233(7): 5133-5141, 2018. PMID: 29226953. DOI: 10.1002/jcp.26395

6 Gershenwald JE, Scolyer RA, Hess KR, Sondak VK, Long GV, Ross MI, Lazar AJ, Faries MB, Kirkwood JM, McArthur GA, Haydu LE, Eggermont AMM, Flaherty KT, Balch CM and Thompson JF; for members of the American Joint Committee on Cancer Melanoma Expert Panel and the International Melanoma Database and Discovery Platform: Melanoma staging: Evidencebased changes in the American Joint Committee on Cancer eighth edition cancer staging manual. CA Cancer J Clin 67(6): 472-492, 2017. PMID: 29028110. DOI: 10.3322/caac.21409

7 Mihic-Probst D, Shea C, Duncan L, de la Fouchardiere A, Landman G, Landsberg J, ven den Oord J, Lowe L, Cook MG, Yun SJ, Clarke L, Messina J, Elder DE and Barnhill RL: Update on Thin Melanoma: Outcome of an International Workshop. Adv Anat Pathol 23: 24-29, 2016. PMID: 26645459. DOI: 10.1097/ PAP.0000000000000100

8 Murali R, Scolyer RA and Thompson JF: Can we better identify thin cutaneous melanomas that are likely to metastasize and cause death? Ann Surg Oncol 19(11): 3310-3312, 2012. PMID: 22777079. DOI: $10.1245 / \mathrm{s} 10434-012-2470-8$

9 Rubinstein JC, Han G, Jackson L, Bulloch K, Ariyan S, Narayan D, Rothberg BG and Han D: Regression in thin melanoma is associated with nodal recurrence after a negative sentinel node biopsy. Cancer Med 5(10): 2832-2840, 2016. PMID: 27671840. DOI: $10.1002 / \mathrm{cam} 4.922$

10 Cherobin ACFP, Wainstein AJA, Colosimo EA, Goulart EMA and Bittencourt FV: Prognostic factors for metastasis in cutaneous melanoma. An Bras Dermatol 93(1): 19-26, 2018. PMID: 29641692. DOI: 10.1590/abd1806-4841.20184779

11 Varamo C, Occelli M, Vivenza D, Merlano M and Lo Nigro C: MicroRNAs role as potential biomarkers and key regulators in melanoma. Genes Chromosomes Cancer 56(1): 3-10, 2017. PMID: 27561079. DOI: $10.1002 / \mathrm{gcc} .22402$

12 Calin GA and Croce CM: MicroRNA signatures in human cancers. Nat Rev Cancer 6(11): 857-866, 2006. PMID: 17060945. DOI: $10.1038 / \mathrm{nrc} 1997$

13 Deng Z, Hao J, Lei D, He Y, Lu L and He L: Pivotal MicroRNAs in Melanoma: A Mini-Review. Mol Diagn Ther 20(5): 449-455, 2016. PMID: 27351922. DOI: 10.1007/s40291-016-0219-y

14 Jayawardana K, Schramm SJ, Tembe V, Mueller S, Thompson JF, Scolyer RA, Mann GJ and Yang J: Identification, review, and systematic cross-validation of microrna prognostic signatures in metastatic melanoma. J Invest Dermatol 136: 245-254, 2016. PMID: 26763444. DOI: 10.1038/JID.2015.355 
15 Glud M and Gniadecki R: MicroRNAs in the pathogenesis of malignant melanoma. J Eur Acad Dermatol Venereol 27: 142-150, 2013. PMID: 22621697. DOI: 10.1111/j.1468-3083.2012.04579.x

16 Segura MF, Greenwald HS, Hanniford D, Osman I and Hernando E: MicroRNA and cutaneous melanoma: from discovery to prognosis and therapy. Carcinogenesis 33: 18231832, 2012. PMID: 22693259. DOI: 10.1093/carcin/bgs205

17 Kozubek J, Ma Z, Fleming E, Duggan T, Wu R, Shin DG and Dadras SS: In-depth characterization of microRNA transcriptome in melanoma. PLoS One 8(9): e72699, 2013. PMID: 24023765. DOI: 10.1371 /journal.pone.0072699

18 Glud M, Rossing M, Hother C, Holst L, Hastrup N, Nielsen FC, Gniadecki R and Drzewiecki KT: Downregulation of miR-125b in metastatic cutaneous malignant melanoma. Melanoma Res 20: 479-484, 2010. PMID: 20827223. DOI: 10.1097/CMR.0b013e32 $833 \mathrm{e} 32 \mathrm{a} 1$

19 Sánchez-Sendra B, Martinez-Ciarpaglini C, González-Muñoz JF, Murgui A, Terrádez L and Monteagudo C: Downregulation of intratumoral expression of miR-205, miR-200c and miR-125b in primary humancutaneous melanomas predicts shorter survival Sci Rep 8(1): 17076, 2018. PMID: 30459436. DOI: 10.1038/ s41598-018-35317-3

20 Riefolo M, Porcellini E, Dika E, Broseghini E and Ferracin M: Interplay between small and long non-coding RNAs in cutaneous melanoma: a complex jigsaw puzzle with missing pieces. Mol Oncol 13(1): 74-98, 2019. PMID: 30499222. DOI: 10.1002/1878-0261.12412

21 Sun V, Zhou WB, Majid S, Kashani-Sabet M and Dar AA: MicroRNA-mediated regulation of melanoma. Br J Dermatol. 171(2): 234-241, 2014. PMID: 24665835. DOI: 10.1111/ bjd.12989

$22 \mathrm{Xu} \mathrm{Y}$, Brenn T, Brown ER, Doherty V and Melton DW: Differential expression of microRNAs during melanoma progression: miR-200c, miR-205 and miR-211 are downregulated in melanoma and act as tumour suppressors. Br J Cancer 106(3): 553-561, 2012. PMID: 22223089. DOI: 10.1038/bjc.2011.568

23 Thompson JF, Soong SJ, Balch CM, Gershenwald JE, Ding S, Coit DG, Flaherty KT, Gimotty PA, Johnson T, Johnson MM, Leong SP, Ross MI, Byrd DR, Cascinelli N, Cochran AJ, Eggermont AM, McMasters KM, Mihm MC Jr., Morton DL and Sondak VK: Prognostic significance of mitotic rate in localized primary cutaneous melanoma: an analysis of patients in the multi-institutional American Joint Committee on Cancer melanoma staging database. J Clin Oncol 29(16): 2199-2205, 2011. PMID: 21519009. DOI: 10.1200/JCO.2010.31.5812

24 Ghanadan A, Ehsani A, Farahmand A and Mirzaei M: Tumor infiltrating lymphocytes in different stages of malignant melanoma and correlation with tumor stage and other prognostic factors: a retrospective multicenter study. Middle East J Cancer 8(4): 207 212, 2017.

25 Dynoodt P, Speeckaert R, De Wever O, Chevolet I, Brochez L, Lambert $\mathrm{J}$ and Van Gele M: miR-145 overexpression suppresses the migration and invasion of metastatic melanoma cells. Int $\mathrm{J}$ Oncol 42: 1443-1451, 2013. PMID: 23404256. DOI: 10.3892/ ijo. 2013.1823

26 Lohcharoenkal W, Das Mahapatra K, Pasquali L, Crudden C, Kular L, Akkaya Ulum YZ, Zhang L, Xu Landén N, Girnita L, Jagodic M, Ståhle M, Sonkoly E and Pivarcsi A: Genome-wide screen for microRNAs reveals a role for miR-203 in melanoma metastasis. J Invest Dermatol 138(4): 882-892, 2018. PMID: 29104160. DOI: $10.1016 /$ j.jid.2017.09.049
27 van Kempen LC, van den Hurk K, Lazar V, Michiels S, Winnepenninckx V, Stas M, Spatz A and van den Oord JJ: Loss of microRNA-200a and c, and microRNA-203 expression at the invasive front of primary cutaneous melanoma is associated with increased thickness and disease progression. Virchows Arch 461(4): 441-448, 2012. PMID: 22956368. DOI: 10.1007/s00428012-1309-9

$28 \mathrm{Bu}$ P and Yang P: MicroRNA-203 inhibits malignant melanoma cell migration by targeting versican. Exp Ther Med 8(1): 309315, 2014. PMID: 24944639. DOI: 10.3892/etm.2014.1708

29 Noguchi S, Mori T, Hoshino Y, Yamada N, Nakagawa T, Sasaki $\mathrm{N}$, Akao Y and Maruo K: Comparative study of anti-oncogenic microRNA-145 in canine and human malignant melanoma. J Vet Med Sci 74(1): 1-8, 2012. PMID: 21836381.

30 Babapoor S, Wu R, Kozubek J, Auidi D, Grant-Kels JM and Dadras SS: Identification of microRNAs associated with invasive and aggressive phenotype in cutaneous melanoma by next-generation sequencing. Lab Invest 97(6): 636-648, 2017. PMID: 28218741. DOI: 10.1038/labinvest.2017.5

31 Liu S, Tetzlaff MT, Liu A, Liegl-Atzwanger B, Guo J and Xu X: Loss of microRNA-205 expression is associated with melanoma progression. Lab Invest 92(7): 1084-1096, 2012. PMID: 2252 5428. DOI: 10.1038/labinvest.2012.62

32 Chang X, Sun Y, Han S, Zhu W, Zhang H and Lian S: MiR-203 inhibits melanoma invasive and proliferative abilities by targeting the polycomb group gene BMI1. Biochem Biophys Res Commun 456(1): 361-366, 2015. PMID: 25475727. DOI: 10.1016/j.bbrc.2014.11.087

33 Montagnani V, Benelli M, Apollo A, Pescucci C, Licastro D, Urso C, Gerlini G, Borgognoni L, Luzzatto L and Stecca B: Thin and thick primary cutaneous melanomas reveal distinct patterns of somatic copy number alterations. Oncotarget 7: 30365-30378, 2016. PMID: 27095580. DOI: 10.18632/oncotarget.8758

34 Hothem Z, Bayci A, Thibodeau BJ, Ketelsen BE, Fortier LE, Uzieblo AF, Cosner D, Totoraitis K, Keidan RD and Wilson GD: Using global gene expression to discriminate thin melanomas with poor outcomes. Mol Cell Oncol 4: e1253527, 2016. PMID: 28197532. DOI: 10.1080/23723556.2016.1253527

35 Richetta AG, Valentini V, Marraffa F, Paolino G, Rizzolo P, Silvestri V, Zelli V, Carbone A, Di Mattia C, Calvieri S, Frascione $\mathrm{P}$, Donati $\mathrm{P}$ and Ottini L: Metastases risk in thin cutaneous melanoma: Prognostic value of clinical-pathological characteristics and mutation profile. Oncotarget 9(63): 32173-32181, 2018. PMID: 30181807. DOI: 10.18632/oncotarget. 25864
Received May 14, 2019

Revised June 11, 2019

Accepted June 12, 2019 